\title{
I-BAND-GEM: a new way for improving BAND-GEM efficiency to thermal and cold neutrons
}

\author{
${\text { Gabriele } \text { Croci }^{1, a} \text {, Andrea Muraro }}^{2}$, Enrico Perelli Cippo ${ }^{2}$, Giovanni Grosso $^{2}$, Carina Höglund $^{3}$, \\ Richard Hall-Wilton ${ }^{3,4}$, Fabrizio Murtas ${ }^{5}$, Davide Raspino ${ }^{6}$, Linda Robinson ${ }^{3}$, Nigel Rhodes ${ }^{6}$, Marica Rebai ${ }^{2}$, \\ Erik Schooneveld ${ }^{6}$, Ilario Defendi ${ }^{7}$, Karl Zeitelhack ${ }^{7}$, Marco Tardocchi ${ }^{2}$, and Giuseppe Gorini ${ }^{1}$ \\ 1 Dipartimento di Fisica "G. Occhialini", Università degli Studi di Milano-Bicocca, Milano, 20125, Italy \\ 2 Istituto di Fisica del Plasma "P. Caldirola" - CNR, Milano, 20125, Italy \\ 3 European Spallation Source ESS AB, Tunavägen 24, 22363 Lund, Sweden \\ 4 Mid-Sweden University, Sundsvall, Sweden \\ ${ }^{5}$ Istituto Nazionale di Fisica Nucleare, Laboratori Nazionali di Frascati, Frascati, 00044, Italy \\ 6 STFC-ISIS Facility, Rutherford Appleton Laboratory, Didcot, UK \\ 7 Heinz Maier-Leibnitz Zentrum (MLZ), TUM, Lichtenbergstr. 1, 85748 Garching, Germany
}

Received: 2 July 2018 / Revised: 8 January 2019

Published online: 19 April 2019

(C) Società Italiana di Fisica / Springer-Verlag GmbH Germany, part of Springer Nature, 2019

\begin{abstract}
The BAND-GEM detector represents one of the novel thermal neutron detection devices that have been developed in order to fulfil the needs of high intensity neutron sources that, like ESS (the European Spallation Source), will start operation in the next few years. The first version of this detector featured a detection efficiency of about $40 \%$ for neutrons with a wavelength of $4 \AA$, a spatial resolution of about $6 \mathrm{~mm}$ and a rate capability in the order of some $\mathrm{MHz} / \mathrm{cm}^{2}$. The novelty of this device is represented by an improved 3D converter cathode $\left(10 \mathrm{~cm}\right.$ thick) based on ${ }^{10} \mathrm{~B}_{4} \mathrm{C}$-coated aluminum grids positioned in a controlled gas mixture volume put on top of a Triple GEM amplifying stage. The position where the neutron interacts in the converter depends on their energy and it was observed that the first version of the detector would suffer from an efficiency decrease for long $(>5 \AA)$ neutron wavelength. This paper describes how the new $3 \mathrm{D}$ cathode allowed improving the detection efficiency at long neutron wavelengths while keeping all the benefits of the first BAND-GEM version.
\end{abstract}

\section{Introduction}

Neutron diffraction and spectroscopy experiments using thermal neutrons are the core activity at spallation neutron sources and up to now detection systems have made great use of ${ }^{3}$ He-based gaseous detectors. The ${ }^{3}$ He shortage [1] and the massive use of this gas in applications linked to homeland security have determined an exponential rise of its price, preventing its use for research applications including future neutron sources such as the European Spallation Source (ESS [2]). In the case of ESS, together with the need for replacing ${ }^{3} \mathrm{He}$, it is essential to develop high-rate neutron detectors [3] able to fully exploit the increased neutron flux of ESS relative to the present neutron sources [4]. The situation called for the development of large area and high-rate neutron detectors with a comparable detection efficiency to those of ${ }^{3} \mathrm{He}$ but with a price ideally not exceeding $500 \mathrm{k} € / \mathrm{m}^{2}$ and a spatial resolution between $1-$ $10 \mathrm{~mm}$. The BAND-GEM (Boron Array Neutron Detector) detector represents one of the established technologies able to fulfill the requirements of new high luminosity spallation sources [5]. This detector is based on the GEM technology [6-9] to detect neutron beams at high rate $\left(>1 \mathrm{MHz} / \mathrm{mm}^{2}\right)$, giving the possibility to cover large areas at low cost. Although GEM-based detectors are mostly used to detect charged particles, they can be adapted (typically by using a customized cathode configuration), to detect neutral particles, such as neutrons and photons [10-13]. Several GEM detectors for fast and thermal neutrons have been realized during the last years [14-33]. The first version of BAND-GEM detector was built and tested in 2016 [34] and although its results were promising, its performance could be further improved especially in the cold neutrons range (wavelength $>5 \AA$ ). This paper describes the construction,

a e-mail: gabriele.croci@unimib.it 


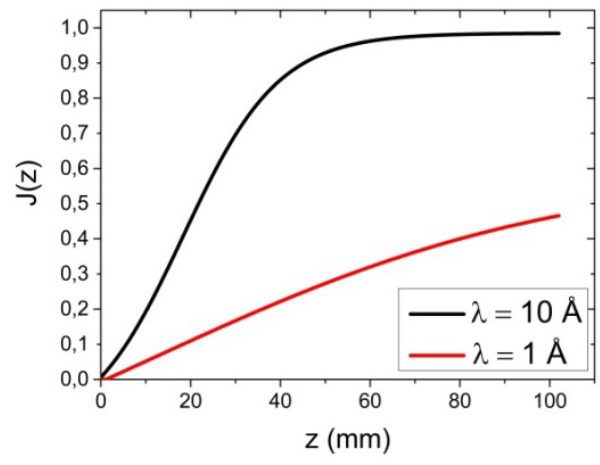

Fig. 1. Neutron conversion probability on the 3D converter for two different wavelengths.

test and results obtained by an improved version of BAND-GEM (named I-BAND-GEM) especially in the detection of long wavelength neutrons.

\section{The I-BAND-GEM principle}

The Boron Array Neutron Detector based on the GEM technology (BAND-GEM) described in [34] consists of a 3D converter cathode mounted on top of a Triple GEM and operated with a $\mathrm{Ar} / \mathrm{CO}_{2} 70 \% / 30 \%$ gas mixture. The 3D converter is made of a stack of aluminum grids coated with $1.1 \mu \mathrm{m}{ }^{10} \mathrm{~B}_{4} \mathrm{C}$ (with boron $99 \%$ isotopically enriched in 10-Boron [35,36]). Each grid is $3 \mathrm{~mm}$ high, has an overall area of $12 \times 7 \mathrm{~cm}^{2}$ and is composed of eleven strips $10 \mathrm{~cm}$ long, $3 \mathrm{~mm}$ high and $200 \mu \mathrm{m}$ thick. The pitch between the strips is $4 \mathrm{~mm}$ and between each couple of grids there are $1 \mathrm{~mm}$ thick fiberglass spacers. Each of the grids is biased with a potential (distributed through a resistor divider) in such a way that the whole 3D cathode acts as a field cage [37]. Three standard GEM foils and a padded anode are placed on top of the borated grid stack. If the full detector is inclined (less than $10^{\circ}$ ) with respect to the incoming beam, neutrons are forced to cross the boron layers at grazing angles and are converted in charged products via the $\mathrm{n}\left({ }^{10} \mathrm{~B},{ }^{7} \mathrm{LI}\right) \alpha$ reaction. As a consequence, the interaction probability, as well as the detection efficiency, is augmented while keeping the beam perturbation small (due to the reduced volume of non-active material). Moreover, conservation of energy and momentum for the nuclear reaction occurring in the layer implies that the two charged particles $\left({ }^{7} \mathrm{Li}\right.$ and $\alpha$ ) are emitted back to back with kinetic energies of the order of $1 \mathrm{MeV}$. It means that at least one of the charged products is likely to leave the ${ }^{10} \mathrm{~B}_{4} \mathrm{C}$ layer and ionize the gas mixture in which the converter is placed. In order to get the signature of the neutron interaction, the primary electrons must be extracted out of the converter, multiplied by the Triple GEM stage and finally read-out by the padded anode. Some of the primary electrons are lost during their path and do not manage to reach the Triple GEM stage because of the particular shape of the 3D converter. The electron extraction efficiency from the neutron converter $(\eta)$ decreases as a linear function of the distance from the Triple GEM [34]. If $\eta$ is too low, the signal can be lost, thus lowering the detection efficiency. This issue is more pronounced for long wavelength neutrons $(>5 \AA)$. Moreover, the ${ }^{10} \mathrm{~B}$ neutron absorption cross section increases linearly with the wavelength of the neutrons (it goes from 2233 barns at $1 \AA$ to 22322 barns at $10 \AA$ ). This increase implies that cold neutrons have a higher probability to convert in the region that is the farthest away from the Triple GEM structure. In order to understand this effect in detail, a numerical simulation was performed. If $p(z)$ represents the neutron absorption probability at a certain depth, its integral $J(z)$,

$$
J(z)=\int_{0}^{z} p\left(z^{\prime}\right) \mathrm{d} z^{\prime},
$$

is directly proportional to the amount of neutrons that will be absorbed up to that specific depth $z$. Figure 1 shows the integral $J(z)$ as a function of $z$ for two different neutron wavelengths, $1 \AA$ and $10 \AA$. In the case of $\lambda=1 \AA$ it is clear that neutrons are absorbed almost uniformly over all the converter depth, while about $80 \%$ of $10 \AA$ neutrons are absorbed for $z<40 \mathrm{~mm}$, i.e. in the converter region far away from the Triple GEM structure. It is thus possible to conclude that neutrons with long wavelength convert mainly in the region were the charge extraction efficiency $\eta$ has its minimum value.

The combination of these two competing effects implies a decrease of overall neutron detection efficiency at long wavelength. The charge extraction efficiency, especially far from the Triple GEM structure, must be augmented, in order to improve the detection efficiency. A single GEM foil was placed in the middle of the neutron converter stack to increase $\eta$ as shown in fig. 2 where both BAND-GEM and I-BAND-GEM design are described. 

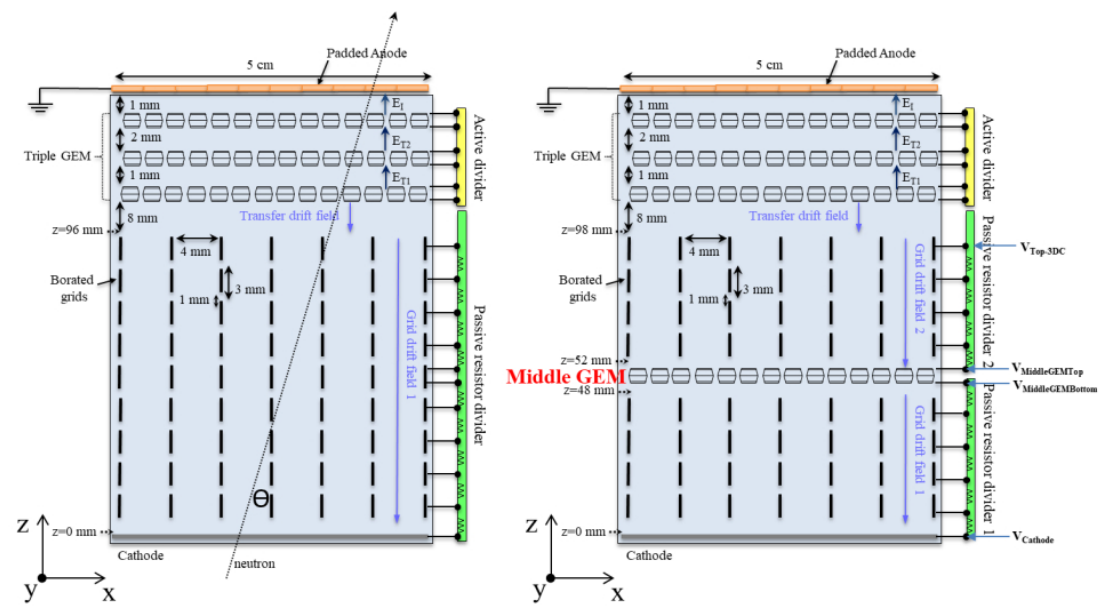

Fig. 2. Layout of BAND-GEM (left) and I-BAND-GEM (right) detectors.
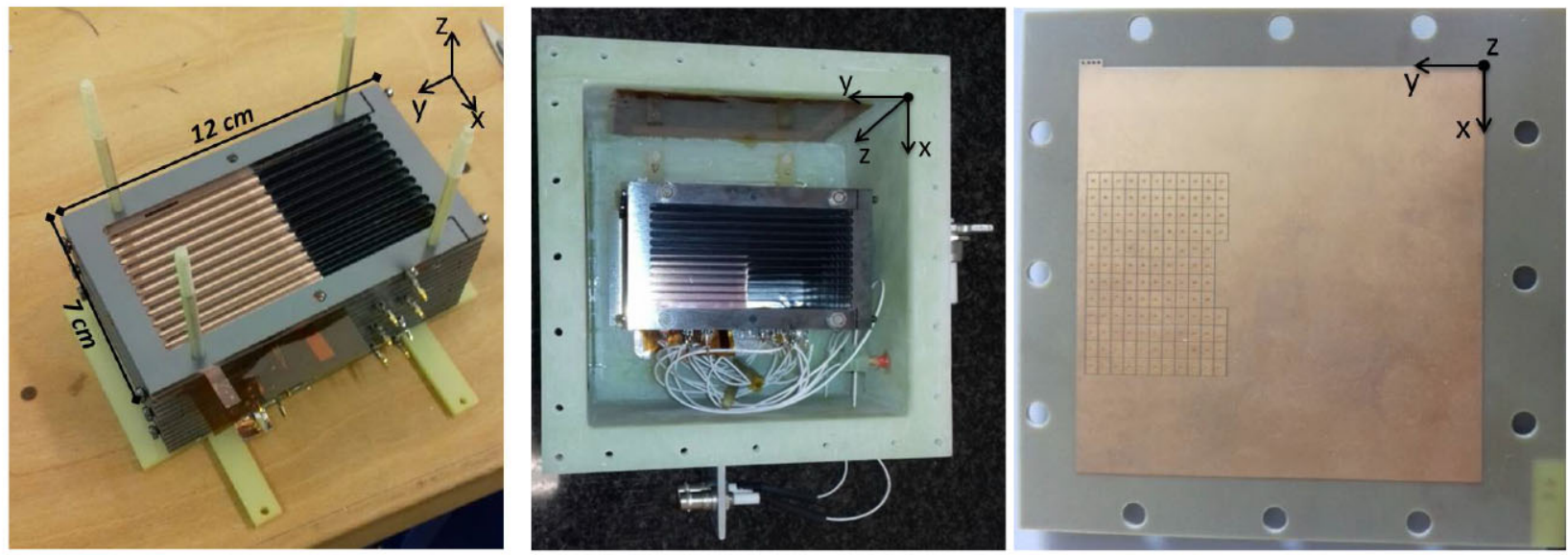

Fig. 3. Pictures of I-BAND-GEM assembly and of the padded anode used to detect signals.

The GEM foil (Middle-GEM) in the middle of the stack, with applied voltage from $250 \mathrm{~V}$ up to maximum $290 \mathrm{~V}$, allows to multiply primary electrons produced close to the cathode by a factor ranging from 1.5 to 3 thus increasing $\eta$ for the region farthest from the Triple GEM and enhancing the overall detection efficiency.

\subsection{I-BAND-GEM construction}

The dimensional and physical parameters of the grids used for the realization of the 3D converter of I-BAND-GEM (see table 1) are the same used for BAND-GEM construction [34].

The main differences are the thickness of the ${ }^{10} \mathrm{~B}_{4} \mathrm{C}$ coating, it was increased to $0.65 \mu \mathrm{m}$ in order to further improve the neutron absorption probability, the presence of the Middle-GEM (that has an active area of $50 \mathrm{~mm} \times 50 \mathrm{~mm}$ ) and the design of the padded anode (compared to the one used in [34]). Figure 3 shows two pictures of the detector assembly and one of the read-out. This is constituted by 128 square pads with an area of $4 \mathrm{~mm}(x$-direction $) \times 3 \mathrm{~mm}$ (y-direction) defining an active area of about $52 \mathrm{~mm}(x) \times 33 \mathrm{~mm}(y)$. The pads were positioned on the side of the detector provided with the Middle-GEM. The signal of each pad is routed to a different channel of a CARIOCA [38] chip whose connector is located on the back of the read-out anode board. The Low Voltage Discriminated Signal (LVDS) signals of each CARIOCA channel are sent to an FPGA motherboard [39] located off the detector. It provides a real time processing of the signal used for the on-line monitoring of the neutron beam. The bias voltage to the three GEM foils is given by means of a custom designed NIM Module (HVGEM) [40] directly interfaced with a control PC. The neutron converter is biased by a module (CAEN A1526 [41]) that is able to deliver up to $15 \mathrm{kV}$. 
Table 1. Geometrical and electrical parameters of the BAND-GEM and the I-BAND-GEM detectors.

\begin{tabular}{|c|c|c|}
\hline & BAND-GEM & I-BAND-GEM \\
\hline Strip pitch & $4 \mathrm{~mm}$ & $4 \mathrm{~mm}$ \\
\hline Grid thickness & $3 \mathrm{~mm}$ & $3 \mathrm{~mm}$ \\
\hline Spacer thickness & $1 \mathrm{~mm}$ & $1 \mathrm{~mm}$ \\
\hline Number of walls per grid & 11 & 11 \\
\hline 3D converter dimensions & $100 \mathrm{~mm}(x) \cdot 50 \mathrm{~mm}(y) \cdot 96 \mathrm{~mm}(z)$ & $100 \mathrm{~mm}(x) \cdot 50 \mathrm{~mm}(y) \cdot 96 \mathrm{~mm}(z)$ \\
\hline Strip thickness & $200 \mu \mathrm{m}$ & $200 \mu \mathrm{m}$ \\
\hline Grid bulk material & Aluminium Al5754 & Aluminium Al5754 \\
\hline $\mathrm{B}_{4} \mathrm{C}$ resisitivity & $696 \Omega \mathrm{cm}$ & $696 \Omega \mathrm{cm}$ \\
\hline${ }^{10} \mathrm{~B}_{4} \mathrm{C}$ thickness on $\mathrm{Al}$ strips & $0.55 \mu \mathrm{m}$ & $0.65 \mu \mathrm{m}$ \\
\hline Tilting angle used for $\eta_{\mathrm{C}}(z)$ measurement & $90^{\circ}$ & $90^{\circ}$ \\
\hline $\begin{array}{l}\text { Tilting angle used for efficiency } \\
\text { and spatial resolution measurements }\end{array}$ & $5^{\circ}$ & $5^{\circ}$ \\
\hline Triple GEM voltage $\left(V_{G E M}\right)$ & $900 \mathrm{~V}$ & $900 \mathrm{~V}$ \\
\hline Induction field $\left(E_{I}\right)$ & $5 \mathrm{kV} / \mathrm{cm}$ & $5 \mathrm{kV} / \mathrm{cm}$ \\
\hline Transfer field $1\left(T_{1}\right)$ & $3 \mathrm{kV} / \mathrm{cm}$ & $3 \mathrm{kV} / \mathrm{cm}$ \\
\hline Transfer field $1\left(T_{2}\right)$ & $3 \mathrm{kV} / \mathrm{cm}$ & $3 \mathrm{kV} / \mathrm{cm}$ \\
\hline Transfer drift field & $1.5 \mathrm{kV} / \mathrm{cm}$ & $1.5 \mathrm{kV} / \mathrm{cm}$ \\
\hline$V_{\text {Top-3DC }}$ & $3.3 \mathrm{kV}$ & $3.3 \mathrm{kV}$ \\
\hline$V_{\text {Middle-GEM-Top }}$ & $1 /$ & $8.5 \mathrm{kV}$ \\
\hline$V_{\text {Middle-GEM-Bottom }}$ & $1 /$ & $8.77 \mathrm{kV}$ \\
\hline$V_{\text {Cathode }}$ & $14.0 \mathrm{kV}$ & $14.0 \mathrm{kV}$ \\
\hline
\end{tabular}

\section{The I-BAND-GEM performance}

\subsection{Experimental Setup at EMMA, CRISP beam lines at ISIS and at the TREFF beam line at FRM2 (include measurements methods)}

The I-BAND-GEM detector prototype was tested at the neutron beam line TREFF at the FRM-II research reactor in Garching (Germany) and at the EMMA and CRISP instruments of the ISIS spallation neutron source in Didcot (United Kingdom). TREFF is a beam line featuring a monochromatic neutron beam with a wavelength of 4.74 $\AA$ [42] and a tunable flux between $10^{3} \mathrm{n} / \mathrm{cm}^{2 *} \mathrm{~s}$ and $10^{6} \mathrm{n} / \mathrm{cm}^{2 *} \mathrm{~s}$. The collimation system allows getting very narrow beam profiles, down to $500 \mu \mathrm{m} \times 500 \mu \mathrm{m}$. The EMMA and CRISP are instruments at the ISIS Target Station 1 [43] and operate at $50 \mathrm{~Hz}$ (EMMA) and $25 \mathrm{~Hz}$ (CRISP), respectively. This difference allows EMMA to have a neutron beam ranging from $1 \AA$ to $4 \AA$ and CRISP from $1 \AA$ to $10 \AA$, each with a maximum flux of about $10^{6} \mathrm{n} / \mathrm{cm}^{2 *} \mathrm{~s}$. This is due to the neutron beam structure in a pulsed source where all neutrons in a bunch are generated at the same $T_{0}$. Long wavelength neutrons have a lower velocity with respect to short wavelength neutrons and thus the time they spend to reach the instrument (e.g., the sample position in CRISP) is longer. The CRISP shutter is designed in order to allow every two bunches to enter the instruments: this allows the possibility to avoid superimposition of long wavelength neutrons belonging to bunch number $i$ with short wavelength neutrons belonging to bunch number $i+1$ that is generated later in time. This implies that CRISP operates at half value of the nominal $50 \mathrm{~Hz}$ frequency, i.e. $25 \mathrm{~Hz}$, but can explore a wider wavelength range.

The aim of the measurements was to determine $\eta$ as a function of converter depth, the overall detection efficiency $\varepsilon$ and the spatial resolution as a function of the neutron wavelength. At TREFF, measurements with a $2 \times 2 \mathrm{~mm}^{2}$ monochromatic beam of $4.74 \AA$ neutrons entering orthogonally with respect to the strips $\left(\theta=90^{\circ}\right)$ allowed to study the $\eta$ of the I-BAND-GEM while measurements with a $4 \times 4 \mathrm{~mm}^{2}$ monochromatic neutron beam entering at $\theta=5^{\circ}$ were done to determine the efficiency of the I-BAND-GEM detector relatively to a calibrated ${ }^{3} \mathrm{He}$ detector. The measurements at ISIS (performed with a $4 \mathrm{~mm} \times 4 \mathrm{~mm}$ beam) were done to determine the detector efficiency as a function of neutron wavelengths between $1 \AA$ and $4 \AA$ (on EMMA [44]) and between $1 \AA$ and $10 \AA$ (on CRISP [43]). 

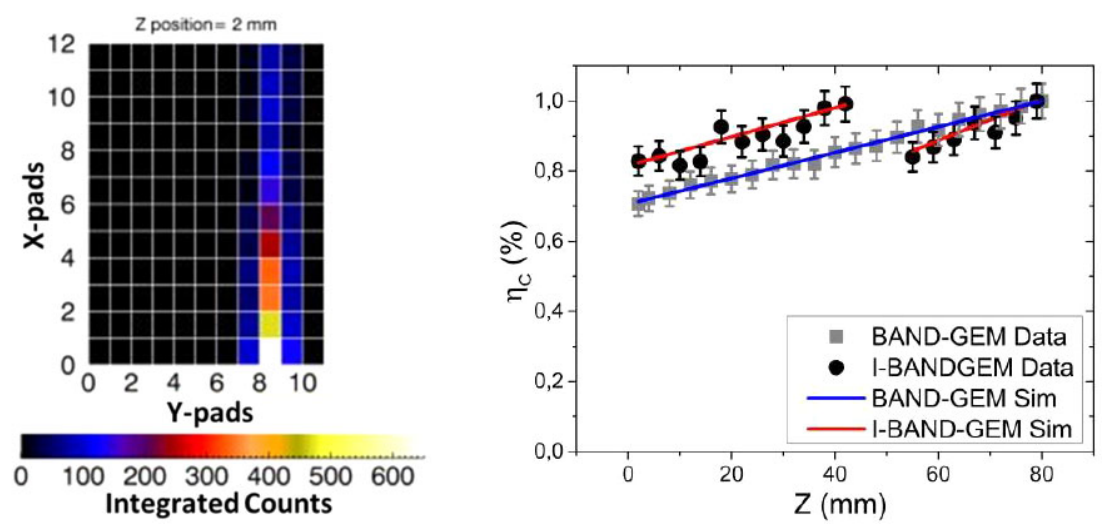

Fig. 4. On the left a footprint of the beam entering the I-BAND-GEM detector at $\Theta=90^{\circ}$ is shown. The color scale represents the integrated counts in 120 seconds. The right panel shows the comparison of relative charge extraction efficiency $\eta_{\mathrm{C}}(z)$ for I-BAND-GEM and BAND-GEM devices. The Middle-GEM was positioned at approximately $z=45 \mathrm{~mm}$ for the I-BAND-GEM only.

At ISIS the efficiency was determined relatively to a standard calibrated beam monitor and using the standard TimeOf-Flight technique as described in [34].

\subsection{Improvement of charge extraction efficiency $\eta_{\mathrm{C}}$}

One of the most important parameters to be determined when working with a $3 \mathrm{D}$ converter that operates also as a field cage is the capability of extracting the primary charge that is released by neutron capture products (either alphas or $\mathrm{Li}$ ions). The electron extraction depends on the applied electric field and the position of the neutron absorption in the field cage. To test the latter dependency, we operated the detector at $90^{\circ}$ with respect to the incoming neutron beam, performing a $z$-direction scan. In this test the neutron are entering in the detector through thin window introduced in the detector only to do this test $(80 \mathrm{~mm} \times 100 \mathrm{~mm}$, with $z=80 \mathrm{~mm}$ the maximum accessible $z$ ). Figure 4 (left) shows how the beam footprint results for one of such measurements at $90^{\circ}$ : the neutron beam (coming from the bottom in the figure) is slowly attenuated along the $y$-direction; the consequent deposited energy shows itself as a long and narrow footprint on the pad plane ( $x$-direction). The integrated (over $300 \mathrm{~s}$ ) counting rate $I_{\mathrm{GEM}}(z)$ was recorded for each $z$-position. The relative charge extraction efficiency $\eta_{\mathrm{C}}$ is defined as $\eta_{\mathrm{C}}(z)=I_{\mathrm{GEM}}(z) / I_{\mathrm{GEM}}(z=80 \mathrm{~mm})$ and shown in fig. 4(right) for both the BAND-GEM and I-BAND-GEM. While for BAND-GEM the curve $\eta_{\mathrm{C}} v s . z$ is linear and decreases with the distance from the Triple GEM (towards negative $z$ ), for I-BAND-GEM the same curve shows a stepwise increase in correspondence of the position of the Middle-GEM (about $z=45 \mathrm{~mm}$ ). The Middle-GEM is enhancing the extraction efficiency: the 3D converter is practically divided in two volumes with approximately the same relative charge extraction efficiency.

Both the results obtained for BAND-GEM and I-BAND-GEM agree with the results of a numerical calculation performed using the ANSYS and Garfield++ software [45]. Ideally $\eta_{\mathrm{C}}$ has to be as close as possible to 1 , providing uniform charge extraction efficiency throughout the depth of the detector.

\subsection{Improvement of neutron detection efficiency}

In order to directly compare the performance of the two detectors, an efficiency measurement of the I-BAND-GEM was performed applying the same method used for the BAND-GEM in [34]. The efficiency of the detector was determined as a function of neutron wavelength in the range accessible on EMMA, namely $1 \AA<\lambda<4 \AA$ m. In addition, for the I-BAND-GEM only, the results of the measurement at TREFF for $\lambda=4.74 \AA$ were added. The tilt angle for both measurements was $\Theta=5^{\circ}$. The comparison of the resulting efficiencies (see fig. 5) shows that I-BAND-GEM has an efficiency of about $10 \%$ higher in this range of wavelengths. This result shows the use of the Middle-GEM implies a better performance, not only at long neutron wavelengths but also at short ones. Also in this case the obtained results are in agreement with the numerical calculation performed using IDL+ANSYS+Garfield++ software [45]. 


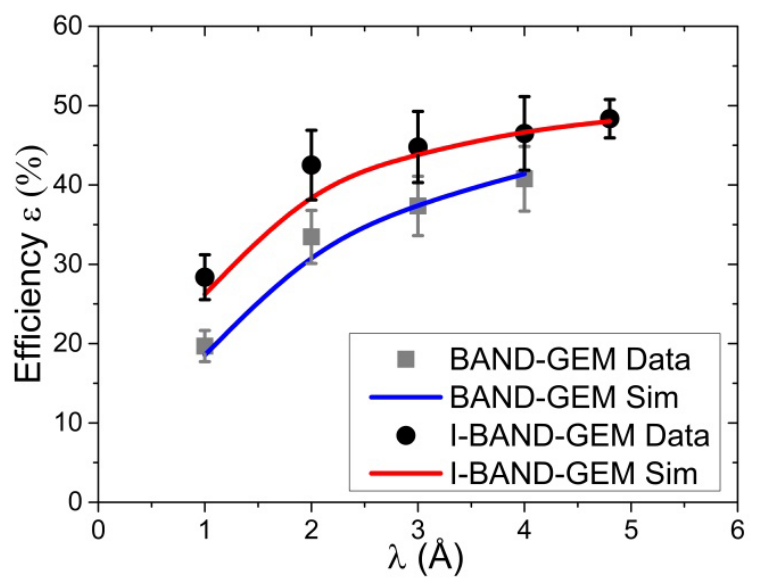

Fig. 5. Comparison of the I-BAND-GEM and BAND-GEM efficiency as a function of neutron wavelengths from $1 \AA$ to $4.74 \AA$, in the simulation and in the experimental data.

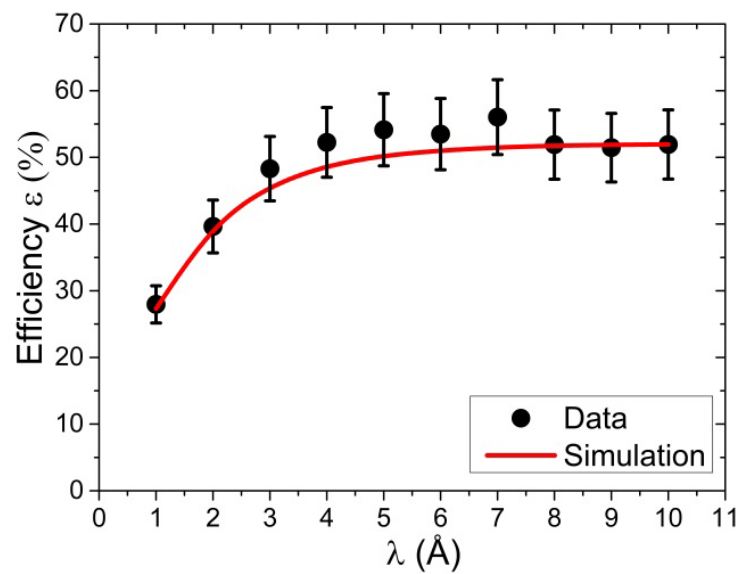

Fig. 6. I-BAND-GEM efficiency as a function of neutron wavelengths from 1 to $10 \AA$, in the simulation and in the experimental data.

\subsection{Measurement of the neutron detection efficiency and the spatial resolution at long neutron wavelengths}

On CRISP we measured the I-BAND-GEM efficiency and spatial resolution at neutron wavelengths in the range between $1 \AA$ and $10 \AA$. The same tilting angle of $\Theta=5^{\circ}$ was set for this measurement too. The efficiency was determined using the Time-Of-Flight technique and relatively to a standard calibrated neutron beam monitor with efficiency of about $0.6 \%$ at $\lambda=1 \AA$ as in [34]. The results are shown in fig. 6 . They show that:

i) up to $5 \AA$ neutrons CRISP results agree with EMMA and TREFF measurements as expected;

ii) the neutron detection efficiency reaches a plateau of about $50 \%$ for $\lambda>5 \AA$. This result is in agreement with the IDL+ANSYS+Garfield ++ simulation. It confirmed that, the increase of the charge extraction efficiency from the 3D-converter volume provide a constant efficiency up to $\lambda=10 \AA$.

The CRISP measurement also allowed determining the spatial resolution of the detector by measuring the FWHM of the reconstructed neutron profile in the two directions, across $(x)$ and along $(y)$ the 3D converter gaps (see fig. 2). The momenta of the spatial neutron distribution were calculated following the same methods described in [34].

The FWHM X measured across the gaps has a larger value (about $6.5 \mathrm{~mm}$ ) than those measured along the gaps, which ranges from 3.5 up to $4.5 \mathrm{~mm}$ (fig. 7). This is due to the fact that the $x$-direction is influenced by the tilting angle that instead does not have any effects on the other direction. The FWHM X shows a slight decrease at long wavelengths. Long wavelength neutrons have a very high probability to interact with the first ${ }^{10} \mathrm{~B}_{4} \mathrm{C}$ layer and as a consequence to give signals whose size is limited to the pads corresponding to a single gap. On the other hand, short wavelength neutrons have a larger probability to give signals on pads corresponding to two or more gaps. The fact that long wavelength neutrons interact with the very first $\mathrm{B}_{4} \mathrm{C}$ layers also implies that the corresponding charged products will release energy in regions close to the cathode: the primary charge has to drift along all the $3 \mathrm{D}$ converter depth 


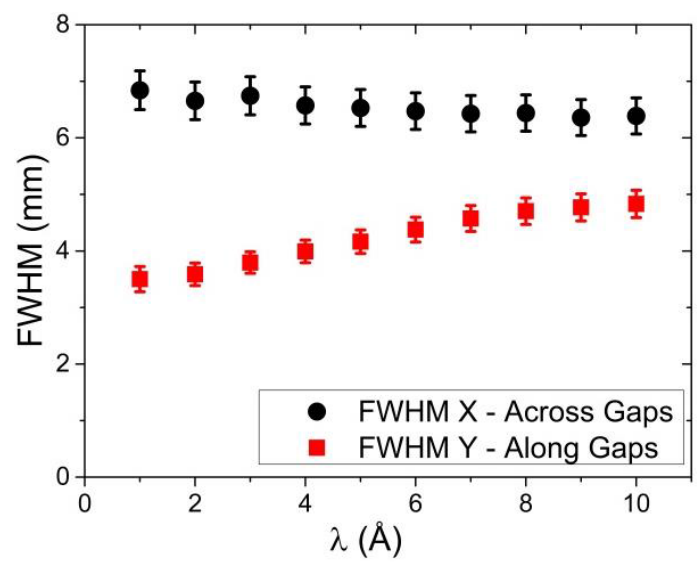

Fig. 7. Measurement of the FWHM across gaps and along gaps of the neutron distribution using I-BAND-GEM. The details of the methods used for FWHM determination are described in [34].

and a higher fraction of the charge is likely to be lost on the grid walls, thus narrowing the whole electronic cloud. The effect on the spatial resolution is a further shrinking of the recorded spot in this direction. On the other hand, the FWHM Y increases at longer wavelengths. This can be explained by considering that the dominant effect in defining the primary cloud dimension in this direction is the transverse diffusion: electron clouds starting close to the cathode will thus experience a larger diffusion than those starting close to the Triple GEM.

\section{Conclusions and future perspective}

The I-BAND-GEM detector described in this paper is one of the most recent developments of neutron detectors based on complex converters: the boron layers are distributed in a number of grids composing a 3D converter, and a proper setting of the field inside the converter, together with the insertion of an amplification stage, ensures good charge collection. A feature of the 3D converter is that, if the whole detector is tilted by an angle $\Theta$ with respect to the direction of incoming neutrons, the thickness of ${ }^{10} \mathrm{~B}_{4} \mathrm{C}$ crossed by the neutrons is increased by a factor $1 / \sin (\Theta)$ so that the neutron "conversion" probability is enhanced and thus the detection efficiency. Moreover, the presence of the Middle-GEM, acting as an electron amplification stage, enhances the charge extraction probability also in the lower efficiency parts of the 3D converter. The detector was tested on the EMMA and CRISP instruments at ISIS and on the TREFF beam line of the FRM-II reactor. A relative charge extraction efficiency across the $3 \mathrm{D}$ converter of $\eta_{\mathrm{C}}=80 \%$ was obtained. This allowed to get efficiency $\varepsilon_{\text {GEM }}$ approaching $50 \%$ at $\lambda>5 \AA \mathrm{m}$ for a tilt angle $\Theta=5^{\circ}$ and neutron energy independent spatial resolution of $\sim 6.5 \mathrm{~mm}$ and $5 \mathrm{~mm}$ across and along the 3D-converter gaps, respectively. These and other features achieved with this new technology make it an attractive candidate for Small Angle Neutron Scattering (SANS) applications. Most of the SANS instruments (e.g., D22 at ILL and Sans2d at ISIS [46]) are nowadays equipped with ${ }^{3} \mathrm{He}$ tubes assuring efficiency larger than $80 \%$, spatial resolution in the order of $5 \mathrm{~mm}$ (depending on specific tube geometry) and rate capability limited to $30-50 \mathrm{kHz} /$ tube. The increase of neutron fluency expected in the future spallation sources (like ESS) joint to the exponential rise of ${ }^{3} \mathrm{He}$ price required development of high rate ${ }^{3} \mathrm{He}$-free detectors as the one described in this paper. The maximum rate achievable with I-BAND-GEM (measured at the Orphee reactor of the LLB-institute in Saclay [47]) was about $1 \mathrm{MHz} / \mathrm{cm}^{2}$. This topic is not treated here since it is the subject of another paper that will be published in the future [48].

The I-BAND-GEM technology is thus one of the candidates for installation (after further optimization) on SANS instruments such as LoKI, one of the first instruments to be installed at the ESS. Further steps will be the construction of a full I-BAND-GEM detector module suitable for installation on LoKI [49]. On the other hand further R\&D work will be performed in the future in order to further increase the detection efficiency: for example the effect of use of more GEM foils in the grid stack will be tested.

We would like to thank A. Balla, G. Corradi and D. Tagnani from the electronic group of Laboratori Nazionali di Frascati (INFN) for all the support about the design of the detector pads and electronics. This work was supported within the CNRSTFC agreement No. 01/9001 concerning collaboration in scientific research at the spallation neutron source ISIS. The financial support of Consiglio Nazionale delle Ricerche (CNR-Italy) is hereby acknowledged. This work was also setup in collaboration and financial support of INFN-group V. Richard Hall-Wilton, Carina Höglund and Linda Robinson would like to acknowledge the financial support of the EU H2020 Brightness Project, grant agreement 676548. All the authors would like to acknowledge colleagues from FRM-II and ISIS for their support and beam-time availability. 


\section{Author contribution statement}

Gabriele Croci, Andrea Muraro, Enrico Perelli Cippo, Giovanni Grosso, Giuseppe Gorini contributed equally to this work.

Publisher's Note The EPJ Publishers remain neutral with regard to jurisdictional claims in published maps and institutional affiliations.

\section{References}

1. Pacific North-West Laboratory, The ${ }^{3}$ He Supply Problem, available at http://www.pnl.gov/main/publications/external/ technical_reports/PNNL-18388.pdf.

2. European Spallation Source, available at http://europeanspallationsource.se/.

3. O. Kirstein et al., PoS (Vertex2014), 029 (2014) arXiv:1411.6194.

4. S. Peggs et al., European Spallation Source Technical Design Report, report 2013-0001, Esss.se.

5. K. Kanaki et al., J. Instrum. 13, P07016 (2018).

6. F. Sauli, Nucl. Instrum. Methods A 386, 531 (1997).

7. S. Duarte Pinto et al., J. Instrum. 4, P12009 (2009).

8. M. Villa et al., Nucl. Instrum. Methods A 628, 182 (2011).

9. M. Alfonsi et al., Nucl. Instrum. Methods A 617, 151 (2010).

10. M. Alexeev et al., Nucl. Instrum. Methods A 610, 174 (2009).

11. M. Alexeev et al., Nucl. Instrum. Methods A 617, 396 (2010).

12. M. Alexeev et al., J. Instrum. 5, P03009 (2010).

13. M. Alexeev et al., Nucl. Instrum. Methods A 623, 129 (2010).

14. M. Rebai et al., Rev. Sci. Instrum. 83, $02 B 721$ (2012).

15. R. Pasqualotto et al., Rev. Sci. Instrum. 83, $02 B 103$ (2012).

16. G. Croci et al., J. Instrum. 7, C03010 (2012).

17. F. Murtas et al., J. Instrum. 7, P07021 (2012).

18. G. Croci et al., Nucl. Instrum. Methods A 720, 144 (2013).

19. G. Croci et al., Nucl. Instrum. Methods A 712, 108 (2013).

20. G. Croci et al., Nucl. Instrum. Methods A 732, 217 (2013).

21. G. Croci et al., EPL 107, 12001 (2014).

22. G. Croci et al., Prog. Theor. Exp. Phys. 2014, $083 \mathrm{H} 01$ (2014).

23. G. Albani et al., J. Instrum. 10, C04040 (2015).

24. G. Croci et al., Eur. Phys. J. Plus 130, 118 (2015).

25. E. Perelli Cippo, G. Croci et al., J. Instrum. 10, P10003 (2015).

26. A. Muraro et al., Nucl. Instrum. Methods A 813, 147 (2016).

27. D. Pfeiffer et al., J. Instrum. 10, P04004 (2015).

28. H. Oshita et al., Nucl. Instrum. Methods A 623, 126 (2010).

29. H. Oshita et al., Nucl. Instrum. Methods A 672, 75 (2012).

30. D. Pfeiffer et al., J. Instrum. 11, P05011 (2016).

31. G. Albani et al., Meas. Sci. Technol. 27, 115902 (2016).

32. M. Köhli et al., Nucl. Instrum. Methods A 828, 242 (2016).

33. M. Henske et al., Nucl. Instrum. Methods A 686, 151 (2012).

34. A. Muraro et al., Prog. Theor. Exp. Phys. 2018, 023H01 (2018).

35. C. Höglund et al., J. Appl. Phys. 111, 104908 (2012).

36. C. Höglund et al., Radiat. Phys. Chem. 113, 14 (2015).

37. B. Ketzer, Nucl. Instrum. A 732, 237 (2013).

38. A. Balla et al., Nucl. Instrum. Methods A 628, 194 (2011).

39. W. Bonivento et al., Nucl. Instrum. Methods A 491, 233 (2002).

40. Design of the FPGA-MB and of HVGEM Module, Gemini LNF Web Site, https://web.infn.it/GEMINI/.

41. http://www . caen. it/csite/CaenProd. jsp?idmod=890\&parent=20.

42. Heinz Maier-Leibnitz Zentrum et al., J. Large-Scale Res. Facil. 3, A121 (2017).

43. https://www.isis.stfc.ac.uk/Pages/crisp-instrument-manual-nov-2010.pdf.

44. T.G. Perring, The resolution function of the chopper spectrometer HET at ISIS, in Journal of Neutron Research, Proceedings of the Twelfth Meeting of the International Collaboration on Advanced Neutron Sources (ICANS-XII) (Cosener's House, Abingdon, UK, 1993) pp. I-328.

45. https://www.harrisgeospatial.com/docs/using_idl_home.html and www.ansys.com; https://cern.ch/garfieldpp.

46. http://www.ill.eu/instruments-support/instruments-groups/instruments/d22/description/instrument-layout/; http://www.isis.stfc.ac.uk/instruments/sans2d/sans2d3000.html.

47. http://www-llb.cea.fr/en/Web/hpr_web/HPRWEB1.php.

48. G. Albani et al., High-rate measurements of the novel BAND-GEM technology for thermal neutron detection at spallation sources, to be submitted to J. Instrum.

49. A.J. Jackson et al., LoKI - A Broad Band High Flux SANS Instrument for the ESS, in Proceedings ICANS XXI Conference, https://doi.org/10.11484/jaea-conf-2015-002. 